\title{
Predictive Value of Exhaled Nitric Oxide and Blood Eosinophil Count in the Assessment of Airway Eosinophilia in COPD
}

This article was published in the following Dove Press journal: International Journal of Chronic Obstructive Pulmonary Disease

\author{
Balazs Antus (iD) ${ }^{1,2}$ \\ Csilla Paska \\ Imre Barta' \\ 'Department of Pathophysiology, \\ National Koranyi Institute of \\ Pulmonology, Budapest $\mathrm{H}-\mathrm{I}$ I2I, Hungary; \\ ${ }^{2}$ Department of Pulmonology, National \\ Koranyi Institute of Pulmonology, \\ Budapest $\mathrm{H}-\mathrm{I}$ I2I, Hungary
}

\begin{abstract}
Purpose: Fractional exhaled nitric oxide $\left(\mathrm{F}_{\mathrm{E}} \mathrm{NO}_{50}\right)$ level and peripheral blood eosinophil count may serve as indicators of airway eosinophilia. The aim of this study was to estimate the diagnostic value of these markers for detecting airway eosinophilia in patients with stable chronic obstructive pulmonary disease (COPD) and those experiencing an acute exacerbation (AECOPD).
\end{abstract}

Patients and Methods: $\mathrm{F}_{\mathrm{E}} \mathrm{NO}_{50}$ levels, sputum and blood eosinophil counts were assessed in 53 clinically stable ex-smoker COPD patients and 67 ex-smoker COPD patients experiencing a severe exacerbation. In AECOPD, clinical variables were measured at the time of hospital admission and discharge following treatment.

Results: In stable COPD, blood eosinophil count but not $\mathrm{F}_{\mathrm{E}} \mathrm{NO}_{50}$ level was found to be a good predictor of airway eosinophilia (area under the receiver operating characteristic curve [ROC AUC]: $\geq 0.82$ ). The sensitivity and the specificity of the test ranged between $75 \%$ and $98 \%$, the negative predictive value (NPV) was high (>90\%). In AECOPD, $\mathrm{F}_{\mathrm{E}} \mathrm{NO}_{50}$ was predictive for airway eosinophilia (ROC AUC: $>0.8$ ) with high NPV $(>88 \%$ ), but with lower sensitivity and specificity (64-70\%). In contrast, the predictive accuracy of blood eosinophil count for airway eosinophilia in AECOPD was modest (ROC AUC: 0.54-0.63). The combined use of the two markers provided only limited additional benefit. Correlation analyses supported ROC curve findings.

Conclusion: In stable COPD the peripheral blood eosinophil count, while in AECOPD the $\mathrm{F}_{\mathrm{E}} \mathrm{NO}_{50}$ level is a good surrogate marker of airway eosinophilia.

Keywords: biomarker, exacerbation, predictive, sensitivity, specificity, surrogate

\section{Introduction}

Several lines of evidence indicate that sputum eosinophil numbers are increased in a subset of patients with chronic obstructive pulmonary disease (COPD), both in stable disease $^{1}$ and acute exacerbation of COPD (AECOPD). ${ }^{2}$ Studies have shown that this subgroup of patients respond favorably to inhaled ${ }^{1}$ or oral corticosteroid treatment, ${ }^{2-5}$ have a reduced airway bacterial load ${ }^{6}$ and exhibit a more pronounced improvement in airflow limitation following treatment of AECOPD $^{7}$ suggesting that they may have a distinct disease phenotype. Therefore, differentiation between eosinophilic and noneosinophilic airway inflammation is of clinical importance.

In recent years both peripheral blood eosinophil count ${ }^{8,9}$ and fractional exhaled nitric oxide $\left(\mathrm{F}_{\mathrm{E}} \mathrm{NO}_{50}\right)$ level ${ }^{10,11}$ have been implicated as surrogate biomarkers to determine and quantify the degree of airway eosinophilia in COPD patients.
Department of Pathophysiology, National Koranyi Institute of Pulmonology, Piheno ut I, Budapest $\mathrm{H}-\mathrm{II} 2 \mathrm{I}$, Hungary

Tel +36 I 39I 3309

Fax + 36 I 2007060

Email antusb@hotmail.com

International Journal of Chronic Obstructive Pulmonary Disease 2020:I5 2025-2035 
However, the predictive accuracy of these markers appears to be variable and often only modest. For example, Negewo et al found that blood eosinophil count can predict airway eosinophilia with a relatively high degree of accuracy in stable COPD patients, ${ }^{12}$ while investigators of the SPIROMICS cohort, a large and well-characterized cohort of COPD patients, concluded that blood eosinophilia alone is not a reliable marker of airway eosinophilia (or phenotyping eosinophil COPD) despite the highly significant correlation between the two measures. ${ }^{13}$

Concerning $\mathrm{F}_{\mathrm{E}} \mathrm{NO}_{50}$, a recent study reported a sensitivity of $62 \%$ and a specificity of $71 \%$ to identify stable COPD patients with eosinophilic airway inflammation. ${ }^{14}$ Our previous data in patients with AECOPD assigned a better discriminatory power to $\mathrm{F}_{\mathrm{E}} \mathrm{NO}_{50} ;{ }^{7}$ however, a more recent study reported less promising data in this respect. ${ }^{15}$ The inconsistent findings could be explained if the diagnostic accuracy of these markers were different between stable disease and AECOPD. Such a scenario is plausible since the local and systemic inflammatory responses, particularly the inflammatory cell ratios differ considerably between the two conditions. ${ }^{16,17}$ Nonetheless, this has not been systematically investigated to date.

Similarly, whether the combination of the two markers has additive value is poorly understood. This is again conceivable since there is evidence that exhaled nitric oxide and blood eosinophils are regulated by distinct inflammatory pathways and could be used as complementary biomarkers to estimate different aspects of an eosinophilic airway inflammation. ${ }^{18}$

Thus, in this prospective study, we (i) determined the predictive accuracy of $\mathrm{F}_{\mathrm{E}} \mathrm{NO}_{50}$ levels and blood eosinophil counts for assessing airway eosinophilia in COPD patients, both in stable disease and AECOPD and (ii) tested whether the combination of these two markers provides additional diagnostic benefit.

\section{Patients and Methods \\ Study Subjects}

Ex-smoker, clinically stable COPD patients and patients hospitalized with AECOPD were recruited for the study between January 2017 and June 2019. Inclusion and exclusion criteria are summarized in Figure 1. Diagnosis of COPD was established by chest physicians and all patients had documented airway obstruction (post-bronchodilator forced expiratory volume in one second $\left[\mathrm{FEV}_{1}\right] /$ forced vital capacity $[\mathrm{FVC}]<0.7)$. AECOPD was defined as increased dyspnea, cough or sputum expectoration (quality or quantity) that led the subject to seek medical attention as specified in international guidelines. ${ }^{19}$ The research

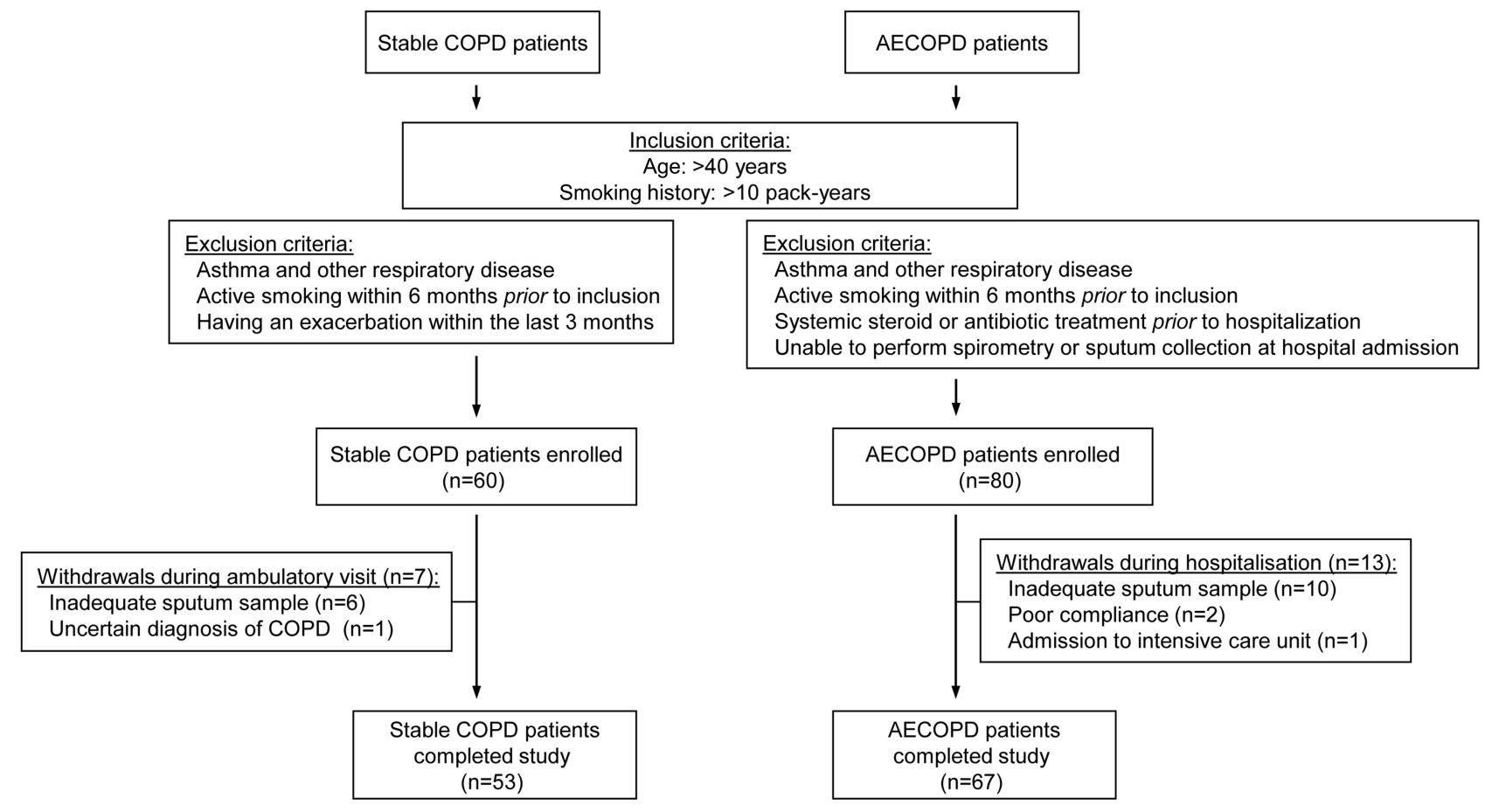

Figure I Flow chart showing the study profile.

Abbreviations: COPD, chronic obstructive pulmonary disease; AECOPD, acute exacerbation of COPD. 
protocol was approved by the Ethics Committee of the National Koranyi Institute of Pulmonology (No: 6/2017), and all subjects gave written informed consent to participate in the study. All procedures performed in the study involving human participants were in accordance with the 1964 Helsinki declaration and its later amendments or comparable ethic standards.

\section{Study Design}

Stable COPD patients provided induced sputum samples during routine clinical visits following lung function, $\mathrm{F}_{\mathrm{E}} \mathrm{NO}_{50}$ and blood gas analysis. From patients with AECOPD spontaneously expectorated sputum was collected, and $\mathrm{F}_{\mathrm{E}} \mathrm{NO}_{50}$, blood gases and lung function parameters were measured at two time points: first at hospital admission, and again on the day of discharge. Routine chemistry and haematology from peripheral venous blood samples were performed on the day of sputum sample collection. Sputum induction, lung function and all other laboratory measurements were performed as previously described., 7,20

\section{Sputum Processing}

Sputum samples were processed in PBS containing dithiothreitol as previously described. ${ }^{7,20}$ Cytospins were stained with May-Grunwald-Giemsa for differential cell counting. At least 400 inflammatory cells were counted for each cytospin slide. The number of inflammatory cells in sputum was recorded as a percentage of total non-squamous cells.

\section{Measurement of $\mathrm{F}_{\mathrm{E}} \mathrm{NO}_{50}$}

$\mathrm{F}_{\mathrm{E}} \mathrm{NO}_{50}$ levels were recorded using a chemiluminescence analyzer (Model LR2500, Logan Research, Rochester, UK) at an exhalation flow rate of $50 \mathrm{~mL} / \mathrm{s}$ according to the procedure recommended by the European Respiratory Society. ${ }^{21}$

\section{Statistical Analysis}

Data are presented as mean \pm SEM or median with interquartile range, as appropriate. Data distribution was analyzed by the Kolmogorov-Smirnov test. Paired Student's $t$-test and the Wilcoxon signed rank test were used to compare variables measured at the time of hospital admission and discharge. Variables between eosinophilic and noneosinophilic patients were analyzed by the unpaired Student's $t$-test or the Mann-Whitney test. Correlation coefficients were calculated by Spearman's method. Receiver operator characteristic (ROC) curve analysis was performed to calculate the predictive accuracy and the optimum cut point of $\mathrm{F}_{\mathrm{E}} \mathrm{NO}_{50}$ and blood eosinophil count for assessing airway eosinophilia. The area under the ROC curve (AUC) was determined, and a value above 0.8 was considered as a good discriminator. $^{22,23}$ Airway eosinophilia was defined as $>2 \%$ sputum eosinophil cell count, but the ROC curve analysis was also performed using the higher cut point of 3\%. Calculations were carried out in GraphPad Prism 4.0 (GraphPad Software Inc., San Diego, CA, USA). A p-value of $<0.05$ was considered significant.

\section{Results}

A total of 60 clinically stable COPD patients and 80 AECOPD patients were enrolled in the study, of which 7 and 13 were withdrawn, respectively, due to their inability to produce adequate sputum samples or unforeseen complications during hospitalization (Figure 1). Demographic and clinical data of the 53 stable COPD and 67 AECOPD patients who completed the study are presented in Tables 1 and 2. Changes in clinical variables during the treatment of AECOPD are provided in Supplementary materials (Tables S1 and S2).

\section{Comparison of Patients with and without Airway Eosinophilia}

Except for blood eosinophil counts, no clinical variables were significantly different between eosinophilic and noneosinophilic patients in the stable COPD group (Table 1).

In contrast, eosinophilic AECOPD patients had higher $\mathrm{F}_{\mathrm{E}} \mathrm{NO}_{50}$ levels but lower C-reactive protein (CRP) levels compared to noneosinophilic patients (Table 2). Additionally, sputum total cell counts, the number and the percentage of sputum neutrophils and the number of lymphocytes were also lower in these subjects.

\section{Correlations Between $\mathrm{F}_{\mathrm{E}} \mathrm{NO}_{50}$, Sputum and Blood Eosinophil Counts}

In stable COPD we found significant positive correlation between sputum eosinophil cell count and the number $(\mathrm{r}=0.557, \mathrm{p}<0.0001)$ and percentage $(\mathrm{r}=0.498, \mathrm{p}<0.001)$ of blood eosinophils. In contrast, $\mathrm{F}_{\mathrm{E}} \mathrm{NO}_{50}$ levels showed no relationship with eosinophil counts in the blood $(r=0.197$, $\mathrm{p}=0.161$ for absolute number and $\mathrm{r}=0.182, \mathrm{p}=0.196$ for percentage $)$ or in the sputum $(\mathrm{r}=0.086, \mathrm{p}=0.554)$.

In AECOPD patients only $\mathrm{F}_{\mathrm{E}} \mathrm{NO}_{50}$ levels and sputum eosinophils showed significant association $(\mathrm{r}=0.362$, $\mathrm{p}=0.003$ ) and neither the number nor the percentage of blood eosinophils were related with either sputum 
Table I Demographic and Clinical Characteristics of Stable COPD Patients

\begin{tabular}{|c|c|c|c|}
\hline & All Stable COPD & $\begin{array}{l}\text { Eosinophilic } \\
\text { COPD }^{\#}\end{array}$ & $\begin{array}{l}\text { Noneosinophilic } \\
\text { COPD }\end{array}$ \\
\hline Subjects $(n)$ & 53 & 13 & 40 \\
\hline Sex (male/female, $n$ ) & $29 / 24$ & $7 / 6$ & $22 / 18$ \\
\hline Age (years) & $65.1 \pm 1.0$ & $67.2 \pm 1.8$ & $64.4 \pm 1.2$ \\
\hline Smoking (pack-years) & $47.8 \pm 3.6$ & $44.7 \pm 6.2$ & $48.9 \pm 4.4$ \\
\hline \multicolumn{4}{|l|}{ GOLD stage $(\mathrm{n}, \%)$} \\
\hline I-II. & $29(55)$ & $9(69)$ & $20(50)$ \\
\hline III-IV. & $24(45)$ & $4(31)$ & $20(50)$ \\
\hline \multicolumn{4}{|c|}{ Inhaled respiratory medication (n, \%) } \\
\hline LAMA or LABA & $4 \mid(77)$ & $12(92)$ & $29(73)$ \\
\hline ICS/LABA & $36(68)$ & $10(77)$ & $26(65)$ \\
\hline BMI $\left(\mathrm{kg} / \mathrm{m}^{2}\right)$ & $27.7 \pm 0.74$ & $25.2 \pm 1.4$ & $28.5 \pm 0.83$ \\
\hline 6MWD (m) & $377.1 \pm 13.4$ & $358.2 \pm 24.7$ & $383.2 \pm 15.9$ \\
\hline \multicolumn{4}{|l|}{ Pulmonary function } \\
\hline FVC (L) & $2.87 \pm 0.14$ & $2.96 \pm 0.27$ & $2.84 \pm 0.16$ \\
\hline FVC (\% predicted) & $73.9 \pm 4.23$ & $78.1 \pm 4.68$ & $72.6 \pm 5.39$ \\
\hline $\mathrm{FEV}_{1}(\mathrm{~L})$ & $1.48 \pm 0.09$ & $1.42 \pm 0.18$ & $1.50 \pm 0.10$ \\
\hline FEV ( $\%$ predicted) & $55.7 \pm 3.09$ & $55.8 \pm 5.55$ & $55.6 \pm 3.72$ \\
\hline $\mathrm{FEV}_{1} / \mathrm{FVC}$ & $0.51 \pm 0.01$ & $0.48 \pm 0.03$ & $0.53 \pm 0.02$ \\
\hline Reversibility (\%) & $10.8 \pm 1.29$ & $10.2 \pm 1.26$ & $12.7 \pm 3.5$ \\
\hline Reversibility (mL) & $153.7 \pm 18.6$ & $152.4 \pm 20.2$ & $157.2 \pm 44.5$ \\
\hline \multicolumn{4}{|l|}{ Blood gases } \\
\hline $\mathrm{PaCO}_{2}(\mathrm{kPa})$ & $5.37 \pm 0.09$ & $5.32 \pm 0.22$ & $5.38 \pm 0.11$ \\
\hline $\mathrm{PaO}_{2}(\mathrm{kPa})$ & $8.32 \pm 0.13$ & $8.30 \pm 0.22$ & $5.38 \pm 0.16$ \\
\hline $\mathrm{F}_{\mathrm{E}} \mathrm{NO}_{50}(\mathrm{ppb})$ & $15.5(9.7-22.0)$ & $20.7(9.2-34.5)$ & $15.5(9.8-20.9)$ \\
\hline \multicolumn{4}{|l|}{ Laboratory data } \\
\hline WBC (cells × $\left.10^{9} / \mathrm{L}\right)$ & $7.8 \mathrm{I} \pm 0.35$ & $8.79 \pm 0.96$ & $7.50 \pm 0.34$ \\
\hline Eosinophils (cells $\times 10^{9} / \mathrm{L}$ ) & $0.17(0.11-0.26)$ & $0.33(0.18-0.85)$ & $0.15(0.1-0.21)^{* *}$ \\
\hline Eosinophils (\%) & $2.0(1.0-4.0)$ & $4.0(2.5-8.5)$ & $2.0(1.0-3.0)^{*}$ \\
\hline CRP (mg/L) & $8.1 \pm 1.1$ & $8.14 \pm 3.08$ & $8.3 \pm 1.04$ \\
\hline \multicolumn{4}{|l|}{ Sputum } \\
\hline Total cell counts $\left(\times 10^{6} / \mathrm{g}\right)$ & $0.96(0.29-2.4)$ & $0.96(0.47-2.5 \mathrm{I})$ & $0.89(0.26-2.35)$ \\
\hline Neutrophils (\%) & $86.6(81.1-92.9)$ & $84.4(70.9-88.8)$ & $88.3(81.5-94.5)$ \\
\hline Neutrophils $\left(\times 10^{6} / \mathrm{g}\right)$ & $0.65(0.25-2.02)$ & $0.69(0.34-1.91)$ & $0.47(0.23-2.02)$ \\
\hline Macrophages (\%) & $6.79(3.25-12.2)$ & $7.03(3.96-9.17)$ & $6.74(3.22-13.6)$ \\
\hline Macrophages $\left(\times 10^{5} / \mathrm{g}\right)$ & $0.55(0.23-1.89)$ & $0.90(0.35-1.90)$ & $0.55(0.1-1.66)$ \\
\hline Lymphocytes (\%) & $3.86(2.2 \mathrm{I}-5.58)$ & $4.95(2.86-5.98)$ & $3.41(2.18-5.28)$ \\
\hline Lymphocytes $\left(\times 10^{4} / g\right)$ & $3.67(1.2-8.08)$ & $5.02(0.20-13.5)$ & $3.42(0.83-7.74)$ \\
\hline Eosinophils (\%) & $0.80(0.0-2.19)$ & $3.15(2.49-7.21)$ & $0.34(0.0-0.98)^{* * *}$ \\
\hline Eosinophils $\left(\times 10^{4} / \mathrm{g}\right)$ & $0.35(0.0-2.33)$ & $3.60(1.88-14.0)$ & $0.043(0.0-0.093)^{* * *}$ \\
\hline
\end{tabular}

Notes: Data are presented as mean \pm SEM or median (interquartile ranges) unless stated otherwise. ${ }^{*}$ Defined as $>2 \%$ sputum eosinophil cell count; ${ }^{*} \mathrm{p}<0.005$, ${ }^{* *} \mathrm{p}<0.001$ and $* * * 00.0001$ vs eosinophilic COPD.

Abbreviations: $\mathrm{FVC}$, forced vital capacity; $\mathrm{FEV}_{1}$, forced expiratory volume in one second; $\mathrm{F}_{\mathrm{E}} \mathrm{NO}_{50}$, fractional exhaled nitric oxide measured at an exhalation flow rate of $50 \mathrm{~mL} / \mathrm{s}$; ppb, parts per billion; 6MWD, 6-min walking distance; $\mathrm{BMI}$, body mass index; $\mathrm{PaCO}_{2}$, arterial carbon dioxide tension; $\mathrm{PaO}$, arterial oxygen tension; GOLD, Global Initiative for Chronic Obstructive Pulmonary Disease; WBC, white blood cell count; CRP, C-reactive protein; ICS, inhaled corticosteroid; LABA, long-acting $\beta_{2}$-agonist; LAMA, long-acting muscarinic antagonist. 
Table 2 Demographic and Clinical Characteristics of AECOPD Patients at the Time of Hospital Admission

\begin{tabular}{|c|c|c|c|}
\hline & All AECOPD & $\begin{array}{l}\text { Eosinophilic } \\
\text { AECOPD }\end{array}$ & $\begin{array}{l}\text { Noneosinophilic } \\
\text { AECOPD }\end{array}$ \\
\hline Subjects (n) & 67 & 15 & 52 \\
\hline Sex (male/female, $n$ ) & $35 / 32$ & $9 / 6$ & $26 / 26$ \\
\hline Age (years) & $69.1 \pm 1.1$ & $68.9 \pm 2.1$ & $69.2 \pm 1.3$ \\
\hline Smoking (pack-years) & $34.5 \pm 3.1$ & $31.5 \pm 7.4$ & $35.3 \pm 3.4$ \\
\hline \multicolumn{4}{|l|}{ GOLD stage (n, \%) } \\
\hline I-II. & $24(36)$ & $7(47)$ & $17(33)$ \\
\hline III-IV. & $43(64)$ & $8(53)$ & $35(67)$ \\
\hline \multicolumn{4}{|c|}{ Inhaled respiratory medication (n, \%) } \\
\hline LAMA or LABA & $64(96)$ & $14(93)$ & $50(96)$ \\
\hline ICS/LABA & $58(87)$ & $12(80)$ & $46(88)$ \\
\hline BMI $\left(\mathrm{kg} / \mathrm{m}^{2}\right)$ & $27.1 \pm 1.3$ & $26.9 \pm 2.4$ & $27.1 \pm 1.6$ \\
\hline \multicolumn{4}{|l|}{ Pulmonary function } \\
\hline FVC (L) & $1.91 \pm 0.08$ & $2.27 \pm 0.19$ & $1.80 \pm 0.09$ \\
\hline FVC (\% predicted) & $64.0 \pm 2.23$ & $69.9 \pm 4.30$ & $62.1 \pm 2.56$ \\
\hline $\mathrm{FEV}_{\mathrm{I}}(\mathrm{L})$ & $0.90 \pm 0.05$ & $1.07 \pm 0.12$ & $0.84 \pm 0.05$ \\
\hline FEV ( $\%$ predicted) & $38.2 \pm 1.85$ & $41.4 \pm 3.91$ & $37.2 \pm 2.10$ \\
\hline $\mathrm{FEV}_{1} / \mathrm{FVC}$ & $0.48 \pm 0.02$ & $0.47 \pm 3.09$ & $0.48 \pm 0.19$ \\
\hline \multicolumn{4}{|l|}{ Blood gases } \\
\hline $\mathrm{PaCO}_{2}(\mathrm{kPa})$ & $5.58 \pm 0.15$ & $5.48 \pm 0.30$ & $5.62 \pm 0.17$ \\
\hline $\mathrm{PaO}_{2}(\mathrm{kPa})$ & $7.10 \pm 0.16$ & $7.84 \pm 0.33$ & $6.88 \pm 0.17^{*}$ \\
\hline $\mathrm{F}_{\mathrm{E}} \mathrm{NO}_{50}(\mathrm{ppb})$ & I4.I (7.I-27.6) & $24.6(14.9-48.5)$ & $11.9(6.3-24.8)^{* *}$ \\
\hline \multicolumn{4}{|l|}{ Laboratory data } \\
\hline WBC (cells × $\left.10^{9} / \mathrm{L}\right)$ & $11.6 \pm 0.7$ & $9.7 \pm 0.9$ & $12.2 \pm 0.84$ \\
\hline Eosinophils (cells $\times 10^{9} / \mathrm{L}$ ) & $0.0(0.0-0.11)$ & $0.09(0.0-0.38)$ & $0.0(0.0-0.09)$ \\
\hline Eosinophils (\%) & $0.0(0.0-1.0)$ & $1.0(0.0-4.0)$ & $0.0(0.0-1.0)$ \\
\hline CRP (mg/L) & $39.9 \pm 7.6$ & $10.7 \pm 4.2$ & $48.3 \pm 9.43^{* *}$ \\
\hline \multicolumn{4}{|l|}{ Sputum } \\
\hline Total cell counts $\left(\times 10^{6} / \mathrm{g}\right)$ & $1.93(0.72-6.81)$ & $0.66(0.34-1.56)$ & $2.76(1.06-7.6)^{* * *}$ \\
\hline Neutrophils (\%) & $93.3(83.3-95.4)$ & $80.6(70.7-84.6)$ & $94.1(87.3-96.2)^{* * *}$ \\
\hline Neutrophils $\left(\times 10^{6} / \mathrm{g}\right)$ & $1.7(0.57-6.4 \mathrm{I})$ & $0.56(0.24-1.16)$ & $2.48(0.9 \mathrm{I}-7.32)^{* * *}$ \\
\hline Macrophages (\%) & $2.14(0.92-5.54)$ & $6.62(2.32-10.9)$ & $1.87(0.68-3.99)^{*}$ \\
\hline Macrophages $\left(\times 10^{5} / \mathrm{g}\right)$ & $0.43(0.16-1.5)$ & $0.37(0.08-0.75)$ & $0.54(0.17-1.55)$ \\
\hline Lymphocytes (\%) & $3.77(1.9-5.62)$ & $4.09(2.07-9.66)$ & $3.62(1.86-5.34)$ \\
\hline Lymphocytes $\left(\times 10^{4} / g\right)$ & $7.6(2.9-25.2)$ & $3.71(1.56-6.52)$ & $9.16(3.56-27.0)^{* *}$ \\
\hline Eosinophils (\%) & $0.0(0.0-1.61)$ & $7.09(2.48-13.6)$ & $0.0(0.0-0.4)^{* * *}$ \\
\hline Eosinophils $\left(\times 10^{4} / g\right)$ & $0.0(0.0-3.31)$ & $4.61(3.43-19.6)$ & $0.0(0.0-1.24)^{* * *}$ \\
\hline
\end{tabular}

Notes: Data are presented as mean \pm SEM or median (interquartile ranges) unless stated otherwise. ${ }^{\#}$ Defined as $>2 \%$ sputum eosinophil cell count; ${ }^{*} \mathrm{p}<0.05$, ${ }^{* *} \mathrm{p}<0.0 \mathrm{I}$ and $* * *$ $p<0.001$ vs eosinophilic COPD.

Abbreviations: $\mathrm{FVC}$, forced vital capacity; $\mathrm{FEV}_{1}$, forced expiratory volume in one second; $\mathrm{F}_{\mathrm{E}} \mathrm{NO}_{50}$, fractional exhaled nitric oxide measured at an exhalation flow rate of $50 \mathrm{~mL} / \mathrm{s}$; ppb, parts per billion; BMI, body mass index; $\mathrm{PaCO}_{2}$, arterial carbon dioxide tension; $\mathrm{PaO}_{2}$, arterial oxygen tension; GOLD, Global Initiative for Chronic Obstructive Pulmonary Disease; WBC, white blood cell count; CRP, C-reactive protein; ICS, inhaled corticosteroid; LABA, long-acting $\beta_{2}$-agonist; LAMA, long-acting muscarinic antagonist.

eosinophils $(\mathrm{r}=0.182, \mathrm{p}=0.15$ and $\mathrm{r}=0.196, \mathrm{p}=0.121$, respectively) or $\mathrm{F}_{\mathrm{E}} \mathrm{NO}_{50}$ levels $(\mathrm{r}=0.205, \mathrm{p}=0.11$ and $\mathrm{r}=0.209, \mathrm{p}=0.104$, respectively) at hospital admission.

At discharge of AECOPD patients neither $\mathrm{F}_{\mathrm{E}} \mathrm{NO}_{50}$ $(r=0.05, \quad p=0.782)$ nor blood eosinophils $(r=0.279$, $\mathrm{p}=0.143$ for absolute and $\mathrm{r}=0.221, \mathrm{p}=0.241$ for percentage) correlated significantly with sputum eosinophils. Similarly, $\mathrm{F}_{\mathrm{E}} \mathrm{NO}_{50}$ levels and blood eosinophils showed no correlation with each other $(r=0.056, p=0.772$ for absolute count and $\mathrm{r}=0.212, \mathrm{p}=0.261$ for percentage). 
A

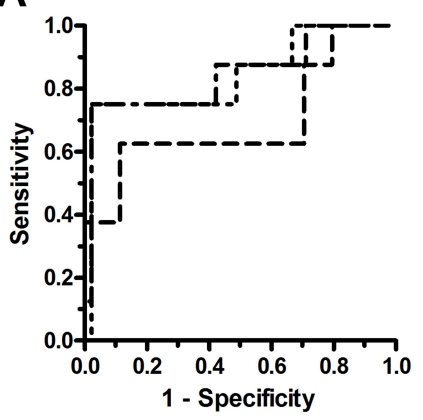

B

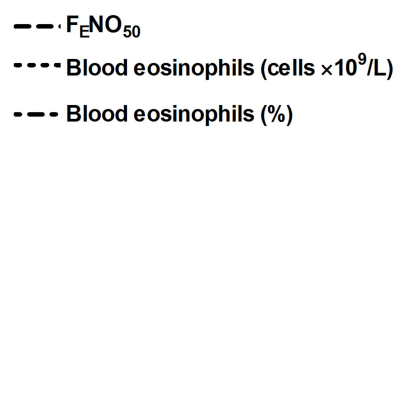

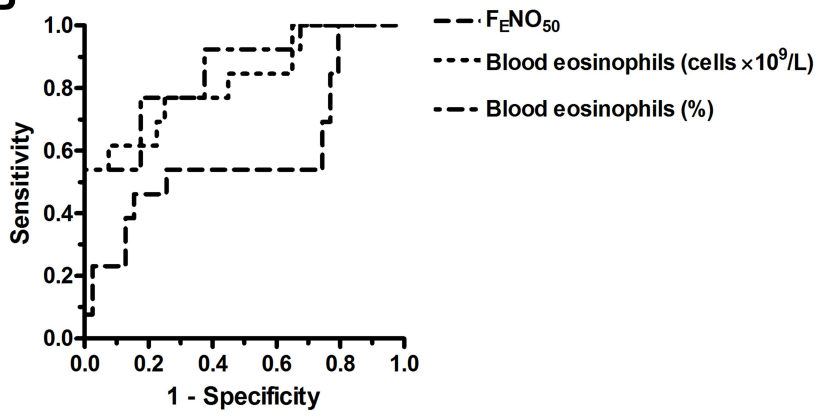

Figure 2 ROC curve for $\mathrm{F}_{\mathrm{E}} \mathrm{NO}_{50}$ and blood eosinophils (absolute count and percentage) to predict airway eosinophilia defined as $>3 \%$ (A) or $>2 \%$ (B) sputum eosinophil cell counts in stable COPD patients.

Predictive Accuracy of $\mathrm{F}_{\mathrm{E}} \mathrm{NO}_{50}$ and Blood Eosinophil Count for Assessing Airway Eosinophilia in Stable COPD

Based on ROC curve analysis, the number of eosinophils in the blood was a strong predictor (ROC AUC: $\geq 0.82$ ) of airway eosinophilia in stable COPD patients, irrespective of whether the cut point of eosinophilia was set at $>3 \%$ or $>2 \%$ (Figure 2, Table 3 ). Notably, at the optimum cut points the negative predictive value (NPV) of the test was high $(>90 \%)$. Although the percentage of blood eosinophils was also a good surrogate marker of airway eosinophilia (ROC AUC: 0.8), its specificity and positive predictive value (PPV) were lower than those of the absolute number.
In contrast to blood eosinophil count, $\mathrm{F}_{\mathrm{E}} \mathrm{NO}_{50}$ was proven to be only a weak predictor (ROC AUC <0.70) of eosinophilic airway inflammation in stable COPD. Interestingly, with airway eosinophilia defined as $>3 \%$ sputum eosinophils, $\mathrm{F}_{\mathrm{E}} \mathrm{NO}_{50}$ had high specificity and NPV. This, however, was associated with low sensitivity and PPV.

\section{Predictive Accuracy of $\mathrm{F}_{\mathrm{E}} \mathrm{NO}_{50}$ and Blood Eosinophil Count for Assessing Airway Eosinophilia in AECOPD}

In AECOPD, neither the absolute number nor the percentage of blood eosinophils was predictive (ROC AUC:

Table 3 Predictive Accuracy of $\mathrm{F}_{\mathrm{E}} \mathrm{NO}_{50}$ and Blood Eosinophil Measurements for Assessing Airway Eosinophilia in Stable COPD Patients

\begin{tabular}{|c|c|c|c|c|c|c|c|c|}
\hline \multicolumn{9}{|l|}{$\mathrm{F}_{\mathrm{E}} \mathrm{NO}_{50}$} \\
\hline $\begin{array}{l}\text { Sputum } \\
\text { Eosinophils }\end{array}$ & AUC & $95 \% \mathrm{Cl}$ & $P$ value & Cut Point (ppb) & Sensitivity (\%) & Specificity (\%) & PPV (\%) & NPV (\%) \\
\hline$>3 \%(n=8)$ & 0.69 & $0.45-0.93$ & 0.097 & 29.5 & 63 & 91 & 56 & 93 \\
\hline$>2 \%(n=13)$ & 0.59 & $0.39-0.79$ & 0.350 & 20.6 & 50 & 75 & 44 & 79 \\
\hline \multicolumn{9}{|c|}{ Blood Eosinophils (absolute count) } \\
\hline $\begin{array}{l}\text { Sputum } \\
\text { Eosinophils }\end{array}$ & AUC & $95 \% \mathrm{Cl}$ & $\mathbf{P}$ value & Cut Point (cells $\left.\times 10^{9} / \mathrm{L}\right)$ & Sensitivity (\%) & Specificity (\%) & $\begin{array}{l}\text { PPV } \\
\text { (\%) }\end{array}$ & $\begin{array}{l}\text { NPV } \\
\text { (\%) }\end{array}$ \\
\hline$>3 \%(n=8)$ & 0.84 & $0.65-1.02$ & 0.003 & 0.316 & 75 & 98 & 86 & 95 \\
\hline$>2 \%(n=13)$ & 0.82 & $0.68-0.96$ & $<0.001$ & 0.204 & 77 & 76 & 53 & 91 \\
\hline \multicolumn{9}{|c|}{ Blood Eosinophils (relative percentage) } \\
\hline $\begin{array}{l}\text { Sputum } \\
\text { Eosinophils }\end{array}$ & AUC & $95 \% \mathrm{Cl}$ & $P$ value & Cut Point (\%) & Sensitivity (\%) & Specificity (\%) & PPV (\%) & NPV (\%) \\
\hline$>3 \%(n=8)$ & 0.80 & $0.58-1.01$ & 0.008 & 3.5 & 75 & 84 & 46 & 95 \\
\hline$>2 \%(n=13)$ & 0.78 & $0.63-0.93$ & 0.003 & 2.5 & 77 & 63 & 42 & 89 \\
\hline
\end{tabular}

Abbreviations: AUC, area under the receiver operating characteristic curve; $\mathrm{Cl}$, confidence interval; $\mathrm{F}_{\mathrm{E}} \mathrm{NO}_{50}$, fractional exhaled nitric oxide measured at an exhalation flow rate of $50 \mathrm{~mL} / \mathrm{s}$; ppb, parts per billion; PPV, positive predictive value; NPV, negative predictive value. 
A

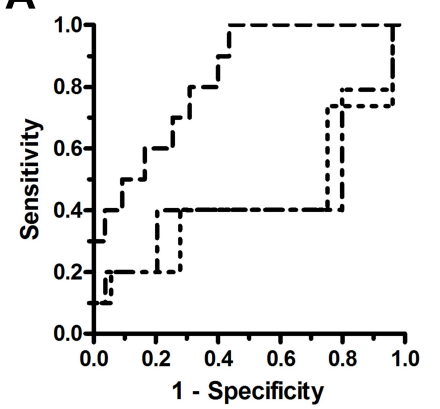

B

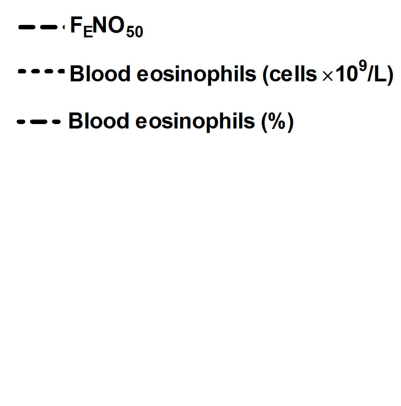

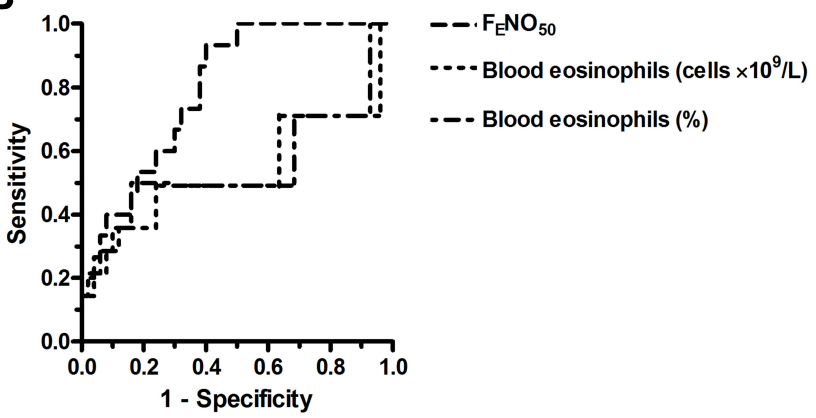

Figure $3 \mathrm{ROC}$ curve for $\mathrm{F}_{\mathrm{E}} \mathrm{NO}_{50}$ and blood eosinophils (absolute count and percentage) to predict airway eosinophilia defined as $>3 \%$ (A) or $>2 \%$ (B) sputum eosinophil cell counts in AECOPD patients.

$<0.65$ ) for airway eosinophilia irrespective of the definition of eosinophilia (Figure 3, Table 4). By contrast, $\mathrm{F}_{\mathrm{E}} \mathrm{NO}_{50}$ had good predictive accuracy (ROC AUC: $\geq 0.8$ ) in these subjects with excellent NPV and relatively good sensitivity and specificity. Of note is, however, that the PPV of the test was low (29-36\%).

ROC curve analysis could not be performed for AECOPD patients at discharge, as none of the patients had $>3 \%$ sputum eosinophil count and only one had $>2 \%$. A summary of the different sensitivity, specificity, PPV and NPV values of $\mathrm{F}_{\mathrm{E}} \mathrm{NO}_{50}$ and blood eosinophil measurements is provided in the Supplementary materials (Table S3).

\section{Combining $\mathrm{F}_{\mathrm{E}} \mathrm{NO}_{50}$ and Blood Eosinophil Measurements for Assessing Airway Eosinophilia}

In stable COPD, using both $\mathrm{F}_{\mathrm{E}} \mathrm{NO}_{50}$ and blood eosinophil values resulted in increased sensitivity but decreased specificity compared to using the blood eosinophil count alone (Table 5). These changes were associated with decreased PPV, particularly, when the cutoff was set at $>3 \%$. The NPV of the two tests combined remained high ( $>92 \%)$.

In AECOPD, the combination of the two measurements resulted in no significant improvement in diagnostic accuracy compared to the $\mathrm{F}_{\mathrm{E}} \mathrm{NO}_{50}$ test alone (Table 5).

Table 4 Predictive Accuracy of $\mathrm{F}_{\mathrm{E}} \mathrm{NO}_{50}$ and Blood Eosinophil Measurements for Assessing Airway Eosinophilia in AECOPD Patients at the Time of Hospital Admission

\begin{tabular}{|c|c|c|c|c|c|c|c|c|}
\hline \multicolumn{9}{|l|}{$\mathrm{F}_{\mathrm{E}} \mathrm{NO}_{50}$} \\
\hline $\begin{array}{l}\text { Sputum } \\
\text { eosinophils }\end{array}$ & AUC & $95 \% \mathrm{Cl}$ & $P$ value & Cut point (ppb) & Sensitivity (\%) & Specificity (\%) & PPV (\%) & NPV (\%) \\
\hline$>3 \%(n=10)$ & 0.83 & $0.7 I-0.95$ & $<0.001$ & 18.7 & 70 & 69 & 29 & 93 \\
\hline$>2 \%(n=15)$ & 0.80 & $0.68-0.91$ & $<0.001$ & 17.5 & 64 & 69 & 36 & 88 \\
\hline \multicolumn{9}{|c|}{ Blood eosinophils (absolute count) } \\
\hline $\begin{array}{l}\text { Sputum } \\
\text { eosinophils }\end{array}$ & AUC & $95 \% \mathrm{Cl}$ & $P$ value & Cut point (cells $\times 10^{9} / \mathrm{L}$ ) & Sensitivity (\%) & Specificity (\%) & PPV (\%) & NPV (\%) \\
\hline$>3 \%(n=10)$ & 0.54 & $0.34-0.75$ & 0.657 & 0.440 & 20 & 94 & 40 & 86 \\
\hline$>2 \%(n=15)$ & 0.63 & $0.45-0.81$ & 0.14 & 0.225 & 40 & 92 & 60 & 83 \\
\hline \multicolumn{9}{|c|}{ Blood eosinophils (relative percentage) } \\
\hline $\begin{array}{l}\text { Sputum } \\
\text { eosinophils }\end{array}$ & AUC & $95 \% \mathrm{Cl}$ & $P$ value & Cut point (\%) & Sensitivity (\%) & Specificity (\%) & PPV (\%) & NPV (\%) \\
\hline$>3 \%(n=10)$ & 0.55 & $0.36-75.5$ & 0.618 & 4.5 & 20 & 94 & 40 & 86 \\
\hline$>2 \%(n=15)$ & 0.63 & $0.45-0.81$ & 0.137 & 4.5 & 21 & 96 & 60 & 81 \\
\hline
\end{tabular}

Abbreviations: $\mathrm{AUC}$, area under the receiver operating characteristic curve; $\mathrm{Cl}$, confidence interval; $\mathrm{F}_{\mathrm{E}} \mathrm{NO}_{50}$, fractional exhaled nitric oxide measured at an exhalation flow rate of $50 \mathrm{~mL} / \mathrm{s}$; ppb, parts per billion; PPV, positive predictive value; NPV, negative predictive value. 
Table 5 Combined Use of $\mathrm{F}_{\mathrm{E}} \mathrm{NO}_{50}$ and Blood Eosinophil Measurements for Assessing Airway Eosinophilia in Stable COPD and AECOPD Patients

\begin{tabular}{|l|l|l|l|l|}
\hline \multicolumn{2}{|l|}{ Stable COPD } & Sensitivity (\%) & Specificity (\%) & PPV (\%) \\
\hline Sputum Eosinophils & 88 & 88 & 58 & NPV (\%) \\
\hline$>3 \%(n=8)$ & 84 & 59 & 42 & 97 \\
$>2 \%(n=13)$ & Sensitivity (\%) & Specificity (\%) & PPV (\%) & 92 \\
\hline AECOPD & 70 & 67 & 29 & NPV (\%) \\
\hline Sputum Eosinophils & 71 & 63 & 36 & 92 \\
\hline$>3 \%(n=10)$ & $72 \%(n=15)$ & 88 & \\
\hline
\end{tabular}

Abbreviations: $\mathrm{F}_{\mathrm{E}} \mathrm{NO}_{50}$, fractional exhaled nitric oxide measured at an exhalation flow rate of $50 \mathrm{~mL} / \mathrm{s}$; PPV, positive predictive value; NPV, negative predictive value.

\section{Discussion}

In this study we compared the diagnostic accuracy of $\mathrm{F}_{\mathrm{E}} \mathrm{NO}_{50}$ level and peripheral blood eosinophil count either used alone or in combination for detecting airway eosinophilia in patients with stable COPD and AECOPD. The main finding of the study was that in stable COPD the blood eosinophil count, while in AECOPD the $\mathrm{F}_{\mathrm{E}} \mathrm{NO}_{50}$ level was predictive for airway eosinophilia. Importantly, the NPV of both tests was high indicating that the clinical significance of using these markers could be the reliable identification of noneosinophilic subjects. The combination of the two markers on the other hand provided limited additional benefit.

As mentioned earlier, measurement of $\mathrm{F}_{\mathrm{E}} \mathrm{NO}_{50}$ may aid the distinction between eosinophilic and noneosinophilic airway inflammation. ${ }^{10,11}$ The measurement used alone ${ }^{24}$ or in combination with blood eosinophil count ${ }^{25}$ has also been implicated in the differential diagnosis between asthma-COPD overlap and COPD, but neither studies involved sputum analysis. The results presented in our study extend the above findings by showing that $\mathrm{F}_{\mathrm{E}} \mathrm{NO}_{50}$ is a strong predictor of airway eosinophilia only in AECOPD, while in stable COPD it has only modest diagnostic value. In agreement with these results we only found a significant correlation between $\mathrm{F}_{\mathrm{E}} \mathrm{NO}_{50}$ levels and sputum eosinophil counts in the AECOPD group.

Although the utility of blood eosinophils in the management of COPD patients, in particular, to guide the use of inhaled corticosteroid (ICS) therapy for exacerbation prevention has been extensively investigated in recent years, ${ }^{8,9}$ the evidence for a relationship between local (airway) and systemic (blood) eosinophilia remains contradictory. ${ }^{12,13}$ We have shown here that the number of eosinophils in the blood is a good indicator of airway eosinophilia in stable
COPD patients but not in those with an ongoing exacerbation where the sensitivity of the test was poor $(20-40 \%)$. Again, the results of the correlation studies supported these findings. Based on our laboratory data we can speculate that the reason why $\mathrm{F}_{\mathrm{E}} \mathrm{NO}_{50}$ and not blood eosinophil count is the better marker of airway eosinophilia in exacerbation is that eosinophilic airway inflammation does not translate to systemic eosinophilia in AECOPD.

The percentage of blood eosinophil count had a slightly lower predictive value compared to the absolute eosinophil count in our study. However, the literature is equivocal whether the percentage or the absolute eosinophil count is more predictive for airway eosinophilia. ${ }^{6,12,13}$ Importantly, the optimum cut points for blood eosinophil count $(0.316$ and 0.204 cells $\times 10^{9} / \mathrm{L}$ ) found in our study were similar to the threshold for eosinophilia (300 cells $/ \mu \mathrm{L}$ ) proposed by the Global Initiative for Chronic Obstructive Lung Disease (GOLD) document. ${ }^{26}$

In patients with AECOPD, Bafadhel and co-workers have shown recently that blood eosinophil count is a sensitive marker of airway eosinophilia (ROC AUC: $0.85),{ }^{2}$ while Gao et al reported that $\mathrm{F}_{\mathrm{E}} \mathrm{NO}_{50}$ is only modestly predictive (ROC AUC: 0.73 ) for eosinophils in sputum. ${ }^{15}$ These findings are in discordance with the observations presented here. We speculate that differences in study populations or the severity of exacerbations could account for the discrepancies between the results. For example, in the study of Gao et $\mathrm{al}^{15}$ smokers were also included in the study population (75\%), even though it is well established that cigarette smoking is an important confounding factor in the measurement of $\mathrm{F}_{\mathrm{E}} \mathrm{NO}_{50} \cdot{ }^{10,11}$ For this very reason, our recruitment strategy called for only ex-smokers, and therefore smoking status did not influence our results. 
In our hands the combination of $\mathrm{F}_{\mathrm{E}} \mathrm{NO}_{50}$ and blood eosinophil measurements provided increased sensitivity but decreased specificity, while the PPV and the NPV did not alter. Thus, it appears that the overall benefit of the combined test is limited. A recent study in asthmatics also failed to detect improvements using the combination of three different eosinophilic biomarkers such as $\mathrm{F}_{\mathrm{E}} \mathrm{NO}_{50}$, blood eosinophils and periostin. ${ }^{27}$

The definition of airway eosinophilia is inconsistent in the literature. When comparing the clinical parameters of our patients, the cut-off value was set at $2 \%$ sputum eosinophil cell count. To allow comparison with other studies, the ROC analysis, as the most important measurement in our study, was performed using a higher (3\%) cut off value as well. Our main findings were independent of the various thresholds set for airway eosinophilia, however, the specificity and the PPV of blood eosinophil count in stable COPD patients considerably improved with the higher cut point ( $>3 \%$ eosinophils in sputum). Nonetheless, irrespective of the cutoff value, both blood eosinophil count and $\mathrm{F}_{\mathrm{E}} \mathrm{NO}_{50}$ (in stable COPD and in AECOPD, respectively) are suitable markers to rule out the presence of eosinophilic airway inflammation with high NPV.

As expected, the clinical characteristics of eosinophilic and noneosinophilic stable COPD patients were similar. By contrast, we observed lower sputum neutrophil count and CRP value in eosinophilic versus noneosinophilic AECOPD patients. These findings are in line with the general view that eosinophilic exacerbations are triggered by viral infections, while neutrophilic inflammation and elevation in systemic inflammatory marker levels may occur in exacerbations due to bacterial infection. ${ }^{6,28,29}$ Nonetheless, the etiology of exacerbations has not been investigated in this study.

ICS therapy has been shown to influence $\mathrm{F}_{\mathrm{E}} \mathrm{NO}_{50}$ levels in some $\mathrm{s}^{30,31}$ but not all ${ }^{32}$ studies. Similarly, the relationship between ICS and blood and/or sputum eosinophil count appears to be ambiguous in COPD patients: some investigators documented no difference between ICS users and non-users ${ }^{6}$ while others found reduced eosinophil counts as a result of ICS treatment. ${ }^{1}$ Retrospective analysis of a clinical trial that compared the effects of various bronchodilators has revealed that ICS has only a small effect on blood eosinophil count in steroid-naïve COPD patients. ${ }^{33}$ In our current study the majority of patients were using ICS, and more importantly the proportion of patients on ICS therapy was similar between eosinophilic and noneosinophilic subjects. Therefore, we speculate that the effect of ICS, if any, would have been comparable in both subgroups of patients. In contrast, any possible confounding effect of systemic corticosteroid therapy on our results can be ruled out, since patients who received systemic steroids before hospitalization were excluded from the study, as indicated on the flow chart (Figure 1).

Stability of $\mathrm{F}_{\mathrm{E}} \mathrm{NO}_{50}$ levels and blood eosinophil counts and the reproducibility of these measurements are important factors that may limit the use of these markers in the management of COPD patients. The issue has been intensively investigated in recent years, particularly the case of blood eosinophils. For example, Landis et al explored the reproducibility of blood eosinophil counts in a large cohort of stable COPD patients over 1 year and concluded that there is good reproducibility, although a subgroup of patients had variable eosinophil counts. ${ }^{34}$ Similarly, Negewo et al investigated the stability of peripheral eosinophil counts between two measurements over a median period of 28 days and found a good agreement between the two measurements. ${ }^{12}$ Nonetheless, other studies documented lower reproducibility and concluded that only a small proportion of patients remain persistently eosinophilic or noneosinophilic over a longer period. ${ }^{35}$ Furthermore, there is evidence that at higher blood eosinophil counts greater variability can be observed. ${ }^{36}$ Concerning $\mathrm{F}_{\mathrm{E}} \mathrm{NO}_{50}$, good repeatability has been reported in several studies ${ }^{37-39}$ although it should be noted that in patients with severe disease difficulties may arise in maintaining the required flow rate and this could impair the repeatability of the measurement. ${ }^{40}$ Nonetheless, the stability of the markers over time has not been investigated in our study.

\section{Conclusion}

In conclusion, our data collected by examination of previously diagnosed COPD patients suggest that surrogate markers to identify airway eosinophilia are not of equal value; instead their value depends on the status of the patient at the time of sample collection. In stable disease it is the peripheral blood eosinophil count, while in acute exacerbation it is the $\mathrm{F}_{\mathrm{E}} \mathrm{NO}_{50}$ level that predicts with good accuracy an increase in the number of eosinophil cells in the sputum. Thus, the difference in the utility of these markers to guide treatment decisions should be considered in clinical practice.

\section{Acknowledgments}

We are grateful to Maria Mikoss and Maria Hernadi (Department of Pathophysiology, National Koranyi 
Institute of Pulmonology) for technical assistance in $\mathrm{F}_{\mathrm{E}} \mathrm{NO}_{50}$ measurement, sputum collection and evaluation. The study was supported by the Hungarian Respiratory Society and the National Research, Development and Innovation Office (OTKA 124343).

\section{Disclosure}

The authors report no conflicts of interest in this work.

\section{References}

1. Leigh R, Pizzichini MM, Morris MM, Maltais F, Hargreave FE, Pizzichini E. Stable COPD: predicting benefit from high-dose inhaled corticosteroid treatment. Eur Respir J. 2006;27:964-971. doi:10. 1183/09031936.06.00072105

2. Bafadhel M, McKenna S, Terry S, et al. Acute exacerbations of COPD: identification of biological clusters and their biomarkers. Am J Respir Crit Care Med. 2011;184:662-671. doi:10.1164/rccm. 201104-0597OC

3. Fujimoto K, Kubo K, Yamamoto H, Yamaguchi S, Matsuzawa Y. Eosinophilic inflammation in the airway is related to glucocorticoid reversibility in patients with pulmonary emphysema. Chest. 1999;115:697-702. doi:10.1378/chest.115.3.697

4. Pizzichini E, Pizzichini MM, Gibson P, et al. Sputum eosinophilia predicts benefit from prednisone in smokers with chronic obstructive bronchitis. Am J Respir Crit Care Med. 1998;158:1511-1517. doi:10.1164/ajrccm.158.5.9804028

5. Brightling CE, Monteiro W, Ward R, Parker D, Morgan MD, Wardlaw AJ. Sputum eosinophilia and short-term response to prednisolone in chronic obstructive pulmonary disease: a randomised controlled trial. Lancet. 2000;356:1480-1485. doi:10.1016/S01406736(00)02872-5

6. Kolsum U, Donaldson GC, Singh R, et al. Blood and sputum eosinophils in COPD; relationship with bacterial load. Respir Res. 2017;18:88. doi:10.1186/s12931-017-0570-5

7. Soter S, Barta I, Antus B. Predicting sputum eosinophilia in exacerbations of COPD using exhaled nitric oxide. Inflammation. 2013;36:1178-1185. doi:10.1007/s10753-013-9653-8

8. Singh D. Predicting corticosteroid response in chronic obstructive pulmonary disease. Blood eosinophils gain momentum. Am J Respir Crit Care Med. 2017;196:1098-1100. doi:10.1164/rccm.20 1703-0592ED

9. Kostikas K, Brindicci C, Patalano F. Blood eosinophils as biomarkers to drive treatment choices in asthma and COPD. Curr Drug Targets. 2018;19:1882-1896. doi:10.2174/1389450119666180212120012

10. Barnes PJ, Dweik RA, Gelb AF, et al. Exhaled nitric oxide in pulmonary diseases: a comprehensive review. Chest. 2010;138: 682-692. doi:10.1378/chest.09-2090

11. Taylor DR, Pijnenburg MW, Smith AD, De Jongste JC. Exhaled nitric oxide measurements: clinical application and interpretation. Thorax. 2006;61:817. doi:10.1136/thx.2005.056093

12. Negewo NA, McDonald VM, Baines KJ, et al. Peripheral blood eosinophils: a surrogate marker for airway eosinophilia in stable COPD. Int J Chron Obstruct Pulmon Dis. 2016;11:1495-1504. doi:10.2147/COPD.S100338

13. Hastie AT, Martinez FJ, Curtis JL, et al. SPIROMICS investigators. Association of sputum and blood eosinophil concentrations with clinical measures of COPD severity: an analysis of the SPIROMICS cohort. Lancet Respir Med. 2017;5:956-967. doi:10.10 16/S2213-2600(17)30432-0

14. Chou KT, Su KC, Huang SF, et al. Exhaled nitric oxide predicts eosinophilic airway inflammation in COPD. Lung. 2014;192: 499-504. doi:10.1007/s00408-014-9591-8
15. Gao J, Zhang M, Zhou L, et al. Correlation between fractional exhaled nitric oxide and sputum eosinophilia in exacerbations of COPD. Int J Chron Obstruct Pulmon Dis. 2017;12:1287-1293. doi: $10.2147 /$ COPD.S134998

16. Fujimoto K, Yasuo M, Urushibata K, Hanaoka M, Koizumi T, Kubo K. Airway inflammation during stable and acutely exacerbated chronic obstructive pulmonary disease. Eur Respir J. 2005;25:640-646. doi:10.1183/09031936.05.00047504

17. Bathoorn E, Liesker JJ, Postma DS, et al. Change in inflammation in out-patient COPD patients from stable phase to a subsequent exacerbation. Int J Chron Obstruct Pulmon Dis. 2009;4:101-109. doi:10.2147/COPD.S4854

18. Çolak Y, Afzal S, Nordestgaard BG, Marott JL, Lange P. Combined value of exhaled nitric oxide and blood eosinophils in chronic airway disease: the Copenhagen General Population Study. Eur Respir J. 2018;52:pii:1800616. doi:10.1183/13993003.00616-2018

19. Rabe KF, Hurd S, Anzueto A, et al. Global initiative for chronic obstructive lung disease. Global strategy for the diagnosis, management, and prevention of chronic obstructive pulmonary disease: GOLD executive summary. Am $J$ Respir Crit Care Med. 2007;176:532-555. doi:10.1164/rccm.200703-456SO

20. Drozdovszky O, Barta I, Antus B. Sputum eicosanoid profiling in exacerbations of chronic obstructive pulmonary disease. Respiration. 2014;87:408-415. doi:10.1159/000358099

21. Horváth I, Barnes PJ, Loukides S, et al. A European respiratory society technical standard: exhaled biomarkers in lung disease. Eur Respir J. 2017;49:1600965. doi:10.1183/13993003.00965-2016

22. Hanley JA, McNeil BJ. The meaning and use of the area under a receiver operating characteristic (ROC) curve. Radiology. 1982;143:29-36. doi:10.1148/radiology.143.1.7063747

23. Fabbri LM, Romagnoli M, Corbetta L, et al. Differences in airway inflammation in patients with fixed airflow obstruction due to asthma or chronic obstructive pulmonary disease. Am J Respir Crit Care Med. 2003;167:418-4124. doi:10.1164/rccm.200203$183 \mathrm{OC}$

24. Chen FJ, Huang XY, Liu YL, Lin GP, Xie CM. Importance of fractional exhaled nitric oxide in the differentiation of asthma-COPD overlap syndrome, asthma, and COPD. Int J Chron Obstruct Pulmon Dis. 2016;11:2385-2390. doi:10.2147/COPD.S115378

25. Takayama Y, Ohnishi H, Ogasawara F, Oyama K, Kubota T, Yokoyama A. Clinical utility of fractional exhaled nitric oxide and blood eosinophils counts in the diagnosis of asthma-COPD overlap. Int J Chron Obstruct Pulmon Dis. 2018;13:2525-2532. doi:10.2147/ COPD.S167600

26. Global Initiative for Chronic Obstructive Lung Disease. Available from: https://goldcopd.org/. Accessed February 19, 2020.

27. Wagener AH, de Nijs SB, Lutter R, et al. External validation of blood eosinophils, $\mathrm{FE}(\mathrm{NO})$ and serum periostin as surrogates for sputum eosinophils in asthma. Thorax. 2015;70:115-120. doi:10.1136/thoraxjnl-2014-205634

28. Kim VL, Coombs NA, Staples KJ, AERIS study group, et al. Impact and associations of eosinophilic inflammation in COPD: analysis of the AERIS cohort. Eur Respir J. 50;2017:1700853. doi:10.1183/ 13993003.00853-2017

29. Papi A, Bellettato CM, Braccioni $F$, et al. Infections and airway inflammation in chronic obstructive pulmonary disease severe exacerbations. Am J Respir Crit Care Med. 2006;173:1114-1121. doi:10.1164/rccm.200506-859OC

30. Högman M, Thornadtsson A, Bröms K, et al. Different relationships between $\mathrm{F}(\mathrm{E}) \mathrm{NO}$ and COPD characteristics in smokers and ex-Smokers. COPD. 2019;16:227-233. doi:10.1080/15412555.2019.1638355

31. Lim CS, Rani FA, Tan LE. Response of exhaled nitric oxide to inhaled corticosteroids in patients with stable COPD: a systematic review and meta-analysis. Clin Respir J. 2018;12:218-226. doi:10.1111/crj.12518 
32. Liu J, Sandrini A, Thurston MC, Yates DH, Thomas PS. Nitric oxide and exhaled breath nitrite/nitrates in chronic obstructive pulmonary disease patients. Respiration. 2007;74:617-623. doi:10.1159/000106379

33. Kreindler JL, Watkins ML, Lettis S, Tal-Singer R, Locantore N. Effect of inhaled corticosteroids on blood eosinophil count in steroidnaïve patients with COPD. BMJ Open Respir Res. 2016;3:e00151. doi:10.1136/bmjresp-2016-000151

34. Landis SH, Suruki R, Hilton E, Compton C, Galwey NW. Stability of blood eosinophil count in patients with COPD in the UK clinical practice research datalink. COPD. 2017;14:382-388. doi:10.1080/ 15412555.2017.1313827

35. Greulich T, Mager S, Lucke T, et al. Longitudinal stability of blood eosinophil count strata in the COPD COSYCONET cohort. Int J Chron Obstruct Pulmon Dis. 2018;13:2999-3002. doi:10.21 47/COPD.S165909

36. Southworth T, Beech G, Foden P, Kolsum U, Singh D. The reproducibility of COPD blood eosinophil counts. Eur Respir J 2018;52:1800427. doi:10.1183/13993003.00427-2018
37. Bhowmik A, Seemungal TA, Donaldson GC, Wedzicha JA. Effects of exacerbations and seasonality on exhaled nitric oxide in COPD. Eur Respir J. 2005;26:1009-1015. doi:10.1183/09031936.05.0004 7305

38. Brindicci C, Ito K, Resta O, Pride N, Barnes PJ, Kharitonov SA. Exhaled nitric oxide from lung periphery is increased in COPD. Eur Respir J. 2005;26:52-59. doi:10.1183/09031936.04.00125304

39. Antus B, Horvath I, Barta I. Assessment of exhaled nitric oxide by a new hand-held device. Respir Med. 2010;104:1377-1380. doi:10.1016/j.rmed.2010.06.005

40. Rouhos A, Kainu A, Piirilä P, et al. Repeatability of exhaled nitric oxide measurements in patients with COPD. Clin Physiol Funct Imaging. 2011;31:26-31. doi:10.1111/j.1475-097X.2010.00975.x

\section{Publish your work in this journal}

The International Journal of COPD is an international, peer-reviewed journal of therapeutics and pharmacology focusing on concise rapid reporting of clinical studies and reviews in COPD. Special focus is given to the pathophysiological processes underlying the disease, intervention programs, patient focused education, and self management protocols. This journal is indexed on PubMed Central, MedLine and CAS. The manuscript management system is completely online and includes a very quick and fair peer-review system, which is all easy to use. Visit http://www.dovepress.com/testimonials.php to read real quotes from published authors. 